\title{
The Mechanics of Gravitation-What It Is; How It Operates
}

\author{
Roger Ellman \\ The-Origin Foundation, Inc., Santa Rosa, USA \\ Email: RogerEllman@The-Origin.org
}

How to cite this paper: Ellman, R. (2017) The Mechanics of GravitationWhat It Is; How It Operates. International Journal of Geosciences, 8, 462-470. https://doi.org/10.4236/ijg.2017.84024

Received: February 7, 2017

Accepted: April 11, 2017

Published: April 18, 2017

Copyright $\odot 2017$ by author and Scientific Research Publishing Inc. This work is licensed under the Creative Commons Attribution International License (CC BY 4.0).

http://creativecommons.org/licenses/by/4.0/

\begin{abstract}
From a start of only the limitation on the speed of light, the necessity of conservation, and the impossibility of an infinity in material reality, the present paper presents a comprehensive development of the mechanics, the operation of gravitation. Experience shows that everything has a cause and that those causes are themselves the results of precedent causes, and ad infinitum. Defining and comprehending the causality or mechanism operating to produce any observed behavior is essential to understanding or explaining the behavior. The behavior of gravitation is well known, described by Newton's Law of Gravitation. But what gravitational mass is, how gravitational behavior comes about, what in material reality produces the effects of gravitation, is little understood. The extant hypotheses include Einstein's General Relativity's bending of space, efforts to develop "quantum gravitation", and attempts to detect "gravitons". None of those addresses the cause, the mechanism of gravitation. As demonstrated in the present and its prior papers, gravitation is an outward flow from gravitating masses. That means that by manipulating that flow gravitation can be controlled. The procedure for obtaining such control and the design for several various applications are presented in the paper Gravitational and Anti-gravitational Applications which is available in this journal.
\end{abstract}

\section{Keywords}

Gravitation, Newton’s “Big G”, Fundamental Constants

\section{Introduction and Summary}

The behavior of gravitation is well known, described by Newton's Law of Gravitation. But what gravitational mass is, how gravitational behavior comes about, what in material reality produces the effects of gravitation, is little understood. The extant hypotheses include Einstein's General Relativity's bending of space, efforts to develop "quantum gravitation", and attempts to detect "gravitons". 
None of those addresses the cause, the mechanism of gravitation.

Experience shows that everything has a cause and that those causes are themselves the results of precedent causes, and ad infinitum. Defining and comprehending the causality or mechanism operating to produce any observed behavior is essential to understanding or explaining the behavior.

From a start of only the limitation on the speed of light, the necessity of conservation, and the impossibility of an infinity in material reality, the present paper presents a comprehensive development of the mechanics, the operation of gravitation

The Einstein model of gravitation is that of his General Theory of Relativity. That theory describes the behavior of gravitation and the effects that it produces. But, it does not address the cause, the mechanism, of that behavior and effects. The closest that General Relativity comes to the cause or mechanism of gravitation is the contending that gravitational mass "curves" or distorts "space", the distortion leading to the various effects of gravitation. However, no cause or mechanism for the contended distortion of space is offered nor is an explanation of what that "space" or space-time is and how it is subject to being curved or distorted.

The comprehensive explanation of the cause and mechanism of gravitation as derived from the origin of the universe, the Modern Newtonian Model of Gravitation, is not a replacement for, nor contradiction of, the useful aspects of General Relativity but a supplementing of it with an alternative model which unlike General Relativity leads to resolution of the problem of "Big G" and to partial control of gravitation and applications of it.

The development of the Modern Newtonian Model of Gravitation consists of the following steps. Each step results in new "hard" facts generated directly from prior "hard" facts. The development does not contain nor rely on opinions. Consequently, while it is deemed a "model" it is an exact factual description of what it treats.

Step 1-How the universe's particles of matter came into existence.

Step 2-How they came to be propagating an outward flow.

Step 3-The reservoir supply for the substance of the outward flow.

Step 4-The speed of the outward flow.

Step 5-A particle's flow encountering another particle slows its outward flow. Step 6-The outward flow has momentum.

Step 7-Gravitation is the momentum reaction to outward flow slowing.

\section{How the Universe's Particles of Matter Came into Existence}

Only absolute nothing [emptiness containing nothing, the zero of existence] requires no explanation of how it came to be. It is to be expected, as the natural condition. The only way something else, a universe, can come into existence from prior absolute nothing without an infinite rate of change at its beginning is to begin as an oscillation of the form $[1-\cos (2 \pi f t)]$. The only way that can 
happen without violating the principle of conservation [no something from nothing and vice versa] is for there simultaneously to have come into existence the negative or opposite of that oscillation $-[1-\cos (2 \pi f t)]$ so that the two together are still equivalent to absolute nothing .

As with Descartes, because we are here thinking about this, then apparently the two oscillations did not promptly mutually annihilate, which means that they were so unstable that they even more promptly exploded into the myriad matter particles of our universe. That myriad particles can themselves only be like their parents, oscillations of the forms $\pm[1-\cos (2 \pi f t)]$.

Each oscillation is three-dimensional because three dimensions is the minimum number that can involve space part of which is not its own boundary. Therefore the oscillations are spherical.

Every oscillation that we know in nature exhibits, and the very theory of oscillations in the abstract requires, that the oscillation consist of two aspects of the substance which is oscillating [e.g. pendulum position and velocity, electric potential and current] storing and exchanging back and forth the energy of the oscillation. With one aspect varying in oscillatory fashion then when that aspect decreases there must be some "place" for its energy to go, a place in which it is stored until it reappears in that aspect when it increases again. It cannot completely disappear or be lost because the oscillation would die. That "place" is the oscillation's second aspect and it obviously must vary in a manner related to the first aspect's variation, but with its energy storage in opposite phase.

The matter of the universe is myriad particles, each a spherical oscillation.

\section{How the Matter Particles Came to Propagating an Outward Flow}

\subsection{Each Matter Particle’s Central Core}

Each of the myriad original matter particle's spherical oscillations had to be of some determined size, some volume. That develops as follows.

Newton's law of gravitation expressed in terms of $m_{\text {source }}$ and $m_{\text {acted-on }}$ and with both sides of the equation divided by $m_{\text {acted-on }}$ is, of course,

$$
a_{\text {grav }}=G \cdot\left[\frac{m_{\text {source }}}{d^{2}}\right]
$$

stating that gravitation is a property of a body's mass.

However, mass and energy are equivalent, so that a mass, $m$, is proportional to a frequency, $f$, that is characteristic of that mass. That is

$$
m \cdot c^{2}=h \cdot f \text { or } f=\left[\frac{c^{2}}{h}\right] \cdot m
$$

so that the $m_{\text {source }}$ of Equation (1) has a corresponding equivalent frequency, $f_{\text {source. }}$

That being the case, the gravitational acceleration, $a_{\text {gram }}$ can be expressed in terms of that frequency as the change, $\Delta V$, in the velocity, $V$, of the attracted mass per time period, $T_{\text {source }}$ of the oscillation at the corresponding frequency, $f_{\text {source }}$ as 
follows.

$$
a_{\text {grav }}=\Delta v / T_{\text {source }}=\Delta v \cdot f_{\text {source }}
$$

It can then be reasoned setting Equation (3) = Equation (1) as follows.

$$
a_{\text {grav }}=\Lambda v \cdot f_{\text {aource }}=G \cdot\left[\frac{m_{\text {source }}}{d^{2}}\right]
$$

Equation (5), below, is obtained by using the fact that frequency is proportional to mass so that with $f_{p}$ and $m_{p}$ as the proton frequency and mass then $f_{\text {source }}=\left[m_{\text {source }} / m_{p}\right] \cdot f_{p}$.

$$
\Lambda v \cdot\left[\frac{m_{\text {source }}}{m_{p}}\right] \cdot f_{p}=G \cdot\left[\frac{m_{\text {source }}}{d^{2}}\right]
$$

Rearranging and canceling $m_{\text {source }}$ on both sides of the equation,

$$
\Lambda v=\frac{G \cdot m_{p}}{d^{2} \cdot f_{p}} \text { per cycle of } f_{\text {source }}
$$

Then substituting, per Equation (2), $m_{p}=\left[h \cdot f_{p}\right] / c^{2}$,

$$
\begin{aligned}
\Lambda \nu & =\left[\frac{G}{d^{2} \cdot f_{p}}\right] \cdot\left[\frac{h \cdot f_{p}}{c^{2}}\right] \\
& =\frac{G \cdot h}{d^{2} \cdot c^{2}} \text { per cycle of } f_{\text {source }}
\end{aligned}
$$

The Planck Length, $l_{P}$ is defined as

$$
l_{P}=\left[\frac{h \cdot G}{2 \pi \cdot c^{3}}\right]^{\frac{1}{2}}
$$

So that $G=\left[\frac{2 \pi \cdot c^{3} \cdot l_{P}^{2}}{h}\right]$

Substituting $G$ as a function of the Planck Length from Equation (8) into $G$ as it is in Equation (7), the following is obtained.

$$
\begin{aligned}
\Lambda v & =\left[\frac{2 \pi \cdot c^{3} \cdot l_{P}^{2}}{h}\right] \cdot\left[\left[\frac{h}{d^{2} \cdot c^{2}}\right]\right] \\
& =c \cdot \frac{2 \pi \cdot l_{P}^{2}}{d^{2}} \text { per cycle of } f_{\text {source }}
\end{aligned}
$$

This result states that:

- the velocity change due to gravitation, $\Delta V$,

- per cycle of the attracting mass's equivalent frequency, $f_{\text {source }}$,

- which quantity, $\Delta v \cdot f_{\text {source }}$ is the gravitational acceleration, $a_{\text {gram }}$

- is a specific fraction of the speed of light, $c$, namely the ratio of:

- $2 \pi$ times the Planck Length squared, $2 \pi \cdot l_{P}^{2}$, to

- the squared separation distance of the masses, $d^{2}$.

That squared ratio is, of course, the usual inverse square behavior.

This also means that at distance $d=\sqrt{2 \pi} \cdot l_{P}$ from the center of the source, attracting mass, the acceleration, $\Delta v$, per cycle of that attracting mass's equiva- 
lent frequency, $f_{\text {source }}$ is equal to the full speed of light, $c$, the most that it is possible to be. In other words, at that [quite close] distance from the source mass the maximum possible gravitational acceleration occurs. That is the significance, the physical meaning, of $l_{P}$ or, rather, of $\sqrt{2 \pi} \cdot l_{P}$.

The physical significance of $\sqrt{2 \pi} \cdot l_{P}$ is that it sets a limit on the minimum separation distance in gravitational interactions and it implies that a "core" of that radius is at the center of fundamental particles having rest mass. That is, Equation (9) clearly implies that it is not possible for a particle having rest mass to be approached closer than that distance.

Having noted at the beginning of this section 2a: "Each of the myriad original matter particle's spherical oscillations had to be of some determined size, some volume" it has now been derived that that volume is a sphere of radius $\sqrt{2 \pi} \cdot l_{P}$.

\subsection{The Outward Flow from Each Matter Particle's Core}

But, the particles' $[1-\cos (2 \pi f t)]$ spherical oscillation in its core volume of radius $\sqrt{2 \pi} \cdot l_{P}$ is not in a container. There is nothing there except the oscillating substance of the oscillation.

Therefore, what "contains" that core's supply of oscillating substance or why doesn't it all just quickly "slosh" out and be gone? The answer is that it is trying to do just that, to "slosh" out, as hard as it can. It cannot help propagating outward because it has no container. But it can only propagate outward at the limiting rate determined by its surface area, $4 \cdot \pi \cdot\left(\sqrt{2 \pi} \cdot l_{P}\right)^{2}$ and the fastest speed possible for it to flow, the speed of light, $c$.

\section{The Reservoir Supply for the Substance of the Outward Flow}

For such a flow to persist and to have persisted the billions of years since the "Big Bang" there must be a supply of that outward flowing substance in every matter particle. And, that "supply" must be an extremely concentrated reservoir of that which flows outward [concentrated relative to the outward flow]. The radius $\sqrt{2 \pi} \cdot l_{P}$ spherical core of each matter particle is the reservoir supplying the billions of years of outward flow propagation from each particle

That the core is impenetrable per Equation (9) is due to its immense concentration of billions of years worth of flow of the outward flow substance in the minute central core of radius $\sqrt{2 \pi} \cdot l_{P}=4.05134 \times 10^{-35}$ meters of every matter particle.

\section{The Speed of the Outward Flow}

The outward flow has to be of the same form as the spherical oscillation that generated it, $[1-\cos (2 \pi f t)]$. It travels outward under the same influence or control as does light: that which we call the permeability and dielectric of free space, $\mu_{0}$ and $\varepsilon_{0}$ and, consequently, the speed of light. The $\mu_{0}$ and $\varepsilon_{0}$, like electric inductance and capacitance, support the two aspects of the oscillation between which the energy exchanges back and forth. 
But, when the original oscillation came into existence it did so in absolute nothing. There was no "free space" with $\mu_{0}$ and $\varepsilon_{0}$. There was nothing but the original oscillation. And, after the immediate explosion into all of the particles of the universe, each of those particles was propagating its outward flow into nothing, emptiness.

where did the $\mu_{0}$ and $\varepsilon_{0}$ come from? The only thing they could have come from was the original oscillation. There is no other possible source because everything else was absolute nothing, "the zero of existence". The $\mu_{0}$ and $\varepsilon_{0}$ are inherent in the substance of the oscillation, which means, $\mu_{0}$ and $\varepsilon_{0}$ are also inherent in the outward propagation. Each particle's outward flow contains its $\mu_{0}$ and $\varepsilon_{0}$.

\section{A Particle's Flow Encountering another Particle Slows Its Outward Flow}

In a universe of the myriad particles resulting from the Big Bang, each of those particles propagating its own outward flow radially in all directions, there are many instances of the flow from one particle [the "source" particle] encountering, running into, the outward-flow-propagating-center of another particle [the "encountered" particle]. Such "source" particle flows are inverse square reduced in magnitude the farther that their wave front has traveled from its "source".

The flow behavior is analogous to that of an electric transmission line where the rate of travel of an oscillation down the line is determined by the time it takes to build up the electric current for each oscillation cycle through each infinitesimal increment of the line's distributed series inductance $\left[L_{p}\right]$ and to build up the electric potential for each oscillation cycle on each infinitesimal increment $\left[C_{p}\right]$ of the line's distributed shunt capacitance. The speed of flow is determined by the well-established electric transmission line relationship equation (10).

$$
\text { Speed [Electric] }=\frac{1}{\sqrt{L_{p} \cdot C_{p}}}
$$

For particles' propagating oscillating flow the factor determining its speed of propagation is the time required to build up the flow amount for each oscillation cycle through each infinitesimal increment of the flow's $\mu_{0}$ [corresponding to $L_{p}$ ] and the flow's potential for each oscillation cycle on each infinitesimal increment of the flow's $\varepsilon_{0}$ [corresponding to $C_{p}$ ]. But, in radially outward propagating particle's flow, the flow amount is inverse square spread out and the potential likewise both in exactly the same proportion as its $\mu_{0}$ and $\varepsilon_{0}$. The ratio of the flow amount to its $\mu_{0}$ and of its flow potential to its $\varepsilon_{0}$ remains constant, and so likewise the speed, radially outward, of its propagation, $c$.

Upon encountering another particle that arriving flow's (scalar not vector) (much inverse square reduced) $\mu_{0}$ and $\varepsilon_{0}$ combine with the (full magnitude) $\mu_{0}$ and $\varepsilon_{0}$ in the new outgoing propagation of the encountered center, the $\mu_{0}$ sum and the $\varepsilon_{0}$ sum each being larger values. The result is that that "encountered" particle's new outward flow is slowed relative to its natural otherwise speed. That 
is, its speed of flow is determined by a combination of the parameters $\mu_{0}$ and $\varepsilon_{0}$ larger than its flow's otherwise natural values. The speed of flow is determined by the well-established relationship

$$
\text { Speed[Electromagnetic] }=\frac{1}{\sqrt{\mu_{0} \cdot \varepsilon_{0}}}
$$

\section{The Outward Flow Has Momentum}

The oscillating substance of each of the myriad particles has, is, causes its mass. There is no other place or thing to be the mass of those particles. Therefore the propagating outward flow has momentum, the inherent effect of the product of mass, inherent in the substance of the flow, and the flow's velocity.

In the absence of other effects the outward flow is naturally radially outward. While the outward flow effectively transmits pulses of momentum outward in its $[1-\cos (2 \pi f t)]$ oscillation, the core source of that flow is experiencing radially inward equal but opposite pulses of momentum in accordance with Newton's third law of motion. In effect the core source is under reaction compression. Because that effect is radially uniform it produces no net affect on the particle.

\section{Gravitation Is the Momentum Reaction to Outward Flow Slowing}

The incoming flow from a distant "source" particle having the effect of slowing the speed of the "encountered" particle's outward propagated flow causes that "encountered" particle's outward flow to have less momentum than if it were not slowed, again momentum being the product of mass and velocity.

Therefore the Newton's Third Law reaction to that reduced outward flow momentum, reaction back on the "encountered" particle, is smaller than otherwise. That effect takes place on the side of the "encountered particle" facing toward the "source" particle from which the slowing-causing flow came.

But, on the opposite side of the "encountered" particle no such slowing of its outward propagated flow is present so that the outward flow has the full natural momentum and the Newton's Third Law reaction on the particle on that side is the full natural amount. Consequently, the "encountered" particle experiencing its usual full momentum reaction back on itself on its side opposite that facing the incoming flow from the "source" but experiencing reduced reaction back on itself on its side facing the incoming flow from the "source", experiences a net momentum reaction toward the "source" particle from which the slowingcausing flow came.

Thus the particle experiences $[1-\cos (2 \pi f t)]$ pulses of momentum increase toward the "source" gravitationally attracting particle which constitute the gravitational acceleration.

\section{Summary and Conclusion}

The Universe's matter came into existence in the only possible form that avoids an impossible infinite rate of change and satisfies the requirement of conserva- 
tion: a pair of oscillations of the form $\pm[1-\cos (2 \pi f t)]$. Witnessed by we here addressing the issue, those did not mutually annihilate so, being unstable, they exploded into the myriad particles of the universe.

Those myriad particles must, inevitably, propagate similar oscillatory outward flow and have been doing so for billions of years.

Their outward flow is at Speed $=\frac{1}{\sqrt{\mu_{0} \cdot \varepsilon_{0}}}$ because the $\mu_{0}$ and $\varepsilon_{0}$ are inherent in its flow.

That particle's outward flow encountering another particle slows the encountered's outward flow on the side encountered because the $\mu_{0}$ and $\varepsilon_{0}$ of the incoming flow add to the $\mu_{0}$ and $\varepsilon_{0}$ of the encountered particle's outward flow so that the encountered's speed of outward flow there is slowed.

That reduces the encountered particle's outward flow momentum there and its reaction back on that side of the encountered particle while on the encountered particle's opposite side the flow and reaction momentum remain the same.

The result is momentum increments accelerating the encountered particle toward the source of the slowing-causing flow, which is gravitational acceleration.

\section{Other}

The foregoing effects are all due to the oscillatory frequency of the momentum-carrying flow and to its being the only source of $\mu_{0}$ and $\varepsilon_{0}$, those all together producing the gravitational effects. But, what about the amplitude of the particles' oscillations and their propagated flows? The details of that are beyond the scope of this paper which is about gravitation.

However, the role of amplitude can be summarized as follows.

1) The \pm polarities of the $\pm[1-\cos (2 \pi f t)]$ oscillations are the \pm charges of the various particles of the universe.

2) The $[1-\cos (2 \pi f t)]$ oscillation propagated wave amplitude delivers pulses of momentum onto any other particles' cores that it encounters.

3) If the source's wave polarity is the same as the encountered wave polarity then the increments of momentum delivered to the encountered particle result in increments of acceleration of it away from, the source of the waves, which is Coulomb repulsion between like charges.

4) If the two particles involved are of opposite polarity then the increments of momentum delivered by the source wave act to cancel or nullify corresponding amounts of increments of momentum in the outgoing pulses of momentum in the propagated flow of the encountered particle. That correspondingly reduces the encountered particle's experienced reaction back onto itself. The encountered particle experiencing reduced reaction on its side facing the source particle and full reaction on the opposite side experiences net increments of acceleration toward the source particle, which is Coulomb opposite charge repulsion.

That constitutes a simple summary of the behavior of Coulomb's Law. For a full development see the paper Inertial Mass, Its Mechanics-What It Is; How It 
Operates [1] and Section 16, A Model for the Universe (6) - The Neutron, Newton's Laws in the book The Origin and Its Meaning'.

For more on gravitation see the book Gravitics-The Physics of the Behavior and Control of Gravitation [2].

\section{Comprehensive Gravitation}

This paper is an essential integral part of a set of papers treating Comprehensive Gravitation. The papers of the set are listed in references [3] [4] [5] [6] and [7].

\section{References* $^{*}$}

[1] Ellman R. (1998) Inertial Mass, Its Mechanics-What It Is; How It Operates, Eprint Archive at http://arXiv.org, physics/9808045.

[2] Ellman, R. (2008) Gravitics-The Physics of the Behavior and Control of Gravitation, The-Origin Foundation, Inc. http://www.The-Origin.org

[3] Ellman, R. (2016) Comprehensive Gravitation. ResearchGate. https://www.researchgate.net/profile/Roger_Ellman/info

[4] Ellman, R. (2016) Connecting Newton's G with the Rest of Physics-Modern Newtonian Gravitation Resolving the Problem of “Big G's" Value. ResearchGate. https://www.researchgate.net/profile/Roger_Ellman/info

[5] Ellman, R. (2016) The Experimental Data Validation of Modern Newtonian Gravitation over General Relativity Gravitation. ResearchGate. https://www.researchgate.net/profile/Roger_Ellman/info

[6] Ellman, R. (2016) The Mechanics of Gravitation-What It Is; How It Operates. ResearchGate. https://www.researchgate.net/profile/Roger_Ellman/info

[7] Ellman, R. (2016) Gravitational and Anti-Gravitational Applications. ResearchGate. https://www.researchgate.net/profile/Roger_Ellman/info

\section{Submit or recommend next manuscript to SCIRP and we will provide best service for you:}

Accepting pre-submission inquiries through Email, Facebook, LinkedIn, Twitter, etc. A wide selection of journals (inclusive of 9 subjects, more than 200 journals)

Providing 24-hour high-quality service

User-friendly online submission system

Fair and swift peer-review system

Efficient typesetting and proofreading procedure

Display of the result of downloads and visits, as well as the number of cited articles

Maximum dissemination of your research work

Submit your manuscript at: http://papersubmission.scirp.org/

Or contact ijg@scirp.org

${ }^{1}$ This paper is based on development in R. Ellman, The Origin and Its Meaning, The-Origin Foundation, Inc., http://www.The-Origin.org, 1997, in which there is more extensive development and the collateral issues are developed.

${ }^{\star}$ References [3]-[7] are a complete set to be taken together. 